\title{
Tratamiento del Pectus excavatum con cirugía minimamente invasiva con la técnica de Donald Nuss.
}

\author{
Treatment of pectus excavatum with minimally invasive surgery by Donald Nuss technique. \\ Mario Lopez-Carranza ${ }^{1}$, Oswaldo Silvia-Rodríguez ${ }^{1}$, Jorge Achata-Arenas ${ }^{2}$, Victor Mendizabal-Quiñonez ${ }^{2}$, \\ Manuel Calvay-Salinas ${ }^{3}$, Herbert Mostacero-Plasencia ${ }^{4}$, Felipe Sandoval-Espinoza ${ }^{5}$, Ricardo Zavaleta-Alfaro ${ }^{5}$
}

\section{RESUMEN}

El Pectus excavatum, es una malformación congénita de la pared anterior del tórax, caracterizada por una depresión del esternón. Objetivo: Evaluar los resultados del tratamiento quirúrgico en pacientes con pectus excavatum, utilizando la técnica de Nuss. Material y métodos: Diez pacientes con Pectus excavatum, de 6 a 22 años, operados, fueron incluidos. El seguimiento fue de 2 a 14 meses. Resultados: La edad promedio fue 11,5 años, ocho fueron varones, seis tuvieron pectus excavatum simétrico, el Índice de Haller promedio fue 5,1. La indicación de cirugía fue sintomatología broncopulmonar en 4, cuatro cardiológica, uno psicológico y otro recidiva de pectus Post-Ravitch. En todos los pacientes se implantó una barra metálica con dos estabilizadores, el tiempo operatorio fue entre 2 y 4 horas y la estancia hospitalaria, 7 días. Tres pacientes presentaron complicaciones postoperatorias: neumotórax en dos y en uno fijación inadecuada; en el seguimiento los pacientes no fueron asintomáticas y todos mostraron satisfacción con el tratamiento. Conclusiones: La técnica de Donald Nuss es un procedimiento que corrige el Pectus Excavatum, reduce el tiempo operatorio y estancia hospitalaria, con presentación de complicaciones menores y resultados clínico-psicológicos satisfactorios.(Rev Med Hered 2011;22:108-114).

PALABRAS CLAVE: Pectus excavatum, técnica de Nuss, toracoscopia, cirugía mínimamente invasiva.

\section{SUMMARY}

Pectus excavatum is a congenital malformation of anterior wall of the chest, characterized by a depression of the sternum. Objective: To evaluate the results of surgical treatment in patients with pectus excavatum, using the technique of Nuss. Material and methods: 10 patients with Pectus excavatum, from 6 to 22 years, were included.Post surgical was follow-up 2 to 14 months. Results: Mean age was 11.5 years, eight were male, six had symmetrical pectus excavatum, the Haller index average 5.1. The indication for surgery was bronchopulmonary, symptons in four, four cardiac, one psychological and another recurrence of pectus Post-Ravitch. All patients received a metal bar with two stabilizers, operative time was between 2 y 4 hours and hospital stay was seven days. Three patients

\footnotetext{
Cirujano de Tórax y Cardiovascular. Hospital Víctor Lazarte Echegaray. ESSALUD. Trujillo, Perú.

Cirujano de Tórax y Cardiovascular. Hospital Nacional Edgardo Rebagliati Martins. ESSALUD. Lima, Perú.

Cirujano de Tórax y Cardiovascular. Hospital Huancayo. ESSALUD. Junin, Perú.

Cirujano General. Hospital Víctor Lazarte Echegaray. ESSALUD. Trujillo, Perú.

Traumatólogo. Hospital Victor Lazarte Echegaray. ESSALUD. Trujillo, Perú.
} 
had postoperative complications: pneumothorax in two and one with inadequate fixation bar; in follow-up patients were asymptomatic and all show satisfaction with treatment. Conclusions: The Donald Nuss technique is a procedure to correct Pectus excavatum, reduces operative time and hospital stay, with presentation of minor complications and clínics and psicológics results.(Rev Med Hered 2011;22:108-114).

KEY WORDS: Pectus excavatum, technical of Nuss, thoracoscopy, minimally invasive surgery.

\section{INTRODUCCIÓN}

El Pectus Excavatum (PE) o pecho hundido, en embudo, de zapatero o infundibuliforme, es una malformación congénita de la pared anterior del tórax, que afecta a los cartílagos costales inferiores, esternón y apéndice xifoides, que provoca una depresión anterior del tórax; es la deformidad torácica mas frecuente con una incidencia de 1 en 400-1000 nacidos vivos y es mas frecuente en hombres en una relación de 4/1. Puede ocasionar compresión de los órganos torácicos provocando dificultad respiratoria, infecciones respiratorias frecuentes, intolerancia al ejercicio, trastornos cardiológicos y psicológicos. Es leve al nacer y más severa en la adolescencia y puede estar asociado a escoliosis, trastornos del tejido conectivo, síndrome de Marfan y Ehlers Danlos (1,2).

La evaluación preoperatoria se ha estandarizada, consiste en elaboración de la historia clínica, radiografía de tórax (RX), tomografía axial computarizada (TAC), prueba de funciones pulmonares y evaluación cardiológica con electrocardiograma y ecocardiograma (1).

El Índice de Haller (IH), evalúa la severidad del PE, es una relación obtenida mediante una TAC, entre el diámetro mayor transversal del tórax y el diámetro menor antero-posterior, medido desde el borde posterior del esternón y el borde anterior del cuerpo vertebral $(1,3)$. La indicación de cirugía incluye por lo menos dos de los siguientes parámetros: progresión de la enfermedad, IH mayor de 3,25, prolapso de la válvula mitral, compresión o desplazamiento del corazón, exámenes de función pulmonar que indican enfermedad restrictiva u obstructiva, recidiva de cirugía abierta (1, 4-6).

Desde 1947 se emplea para la corrección quirúrgica la técnica de Ravitch o sus modificaciones. Es una cirugía abierta con una incisión amplia en el tórax anterior transversal o longitudinal, con resecciones de 3 o más cartílagos costales a cada lado y sección total o parcial del esternón. En 1998 Donald Nuss, reporta la técnica minimamente invasiva, con incisiones laterales de $2,5 \mathrm{~cm}$. en el tórax e implantación de una barra metálica por vía retroesternal, guiada por videotoracoscopia. La edad adecuada es de 6 a 14 años debido a que la pared torácica es más maleable. Donald Nuss basó su técnica en la experiencia de los ortopedistas en el tratamiento del equinovarus, de las deformidades de la columna vertebral y de los procedimientos ortodóncicos; concluyendo que la fijación del esternón en una posición adecuada podría reconstruir o remodelar los cartílagos costales y conservar la anatomía normal (5-10).

El objetivo del estudio fue evaluar los resultados del tratamiento quirúrgico en pacientes con pectus excavatum, utilizando la técnica de Nuss.

\section{MATERIAL Y MÉTODOS}

Estudio retrospectivo, de tipo serie de casos. Se incluyeron 10 pacientes con $\mathrm{PE}$, a quienes se les realizó corrección quirúrgica empleando la técnica de Donald Nuss, minimamente invasiva por vía toracoscópica en los hospitales de ESSALUD, 5 pacientes en el Hospital "Víctor Lazarte Echegaray" de Trujillo, 3 en el Hospital de Huancayo y 2 en el Hospital Nacional "Edgardo Rebagliati Martins" de Lima, entre marzo 2009 a marzo de 2010.

A todos los pacientes se les realizó una historia clínica, evaluación neumológica, cardiológica y psicológica, RX y TAC de tórax para evaluación de Índice Haller. Las variables estudiadas, fueron: edad, sexo, manifestaciones clínicas, tipo de PE, determinación del Î́ndice de Haller, tiempo quirúrgico, estancia postoperatoria, complicaciones y seguimiento.

\section{Técnica quirúrgica:}

Bajo anestesia general intubada e instalación de catéter epidural para control analgésico, se realizaron mediciones en la pared del tórax para seleccionar la 
barra metálica, la que se moldea en forma curva, según el paciente. Se realizaron incisiones laterales transversales de $2-3 \mathrm{~cm}$. desde la línea axilar anterior hacia atrás. En la incisión lateral derecha se introdujo la pinza diseñada por Kelly, guiada con videotoracoscopía disecando el espacio virtual entre el esternón y el pericardio, saliendo por el lado izquierdo; en la punta de la pinza se anudó una cinta y luego se retiró hacia el lado derecho. La cinta guía se ató a un extremo de la barra moldeada y en posición cóncava fue jalada por la guía en la cavidad pleural y el espacio virtual entre el esternón y el pericardio, saliendo por el lado izquierdo. Una vez instalada la barra adecuadamente se dio un giro de 180 grados con volteadores especiales, de tal manera que la barra quedo en forma convexa elevando el esternón. En los extremos de la barra se colocó un estabilizador para cada lado, fijándose a la costilla subyacente con alambre de acero inoxidable o nylon. El control del dolor se realizó inicialmente con analgesia por el catéter epidural, luego por vía endovonesa y oral.

A los pacientes se les realizó seguimiento de 2 a 14 meses, evaluando aspectos clínicos, como patología broncopulmonar y cardiológica, complicaciones tardías y satisfacción de los pacientes, determinado por mejoría en calidad de vida e imagen corporal.

\section{RESULTADOS}

La edad promedio fue 11,8 (rango: 6 - 22 años); 8 de sexo masculino. El PE fue simétrico en 6 pacientes (Figura 1) y asimétrico en 4. El IH promedio fue 5,1

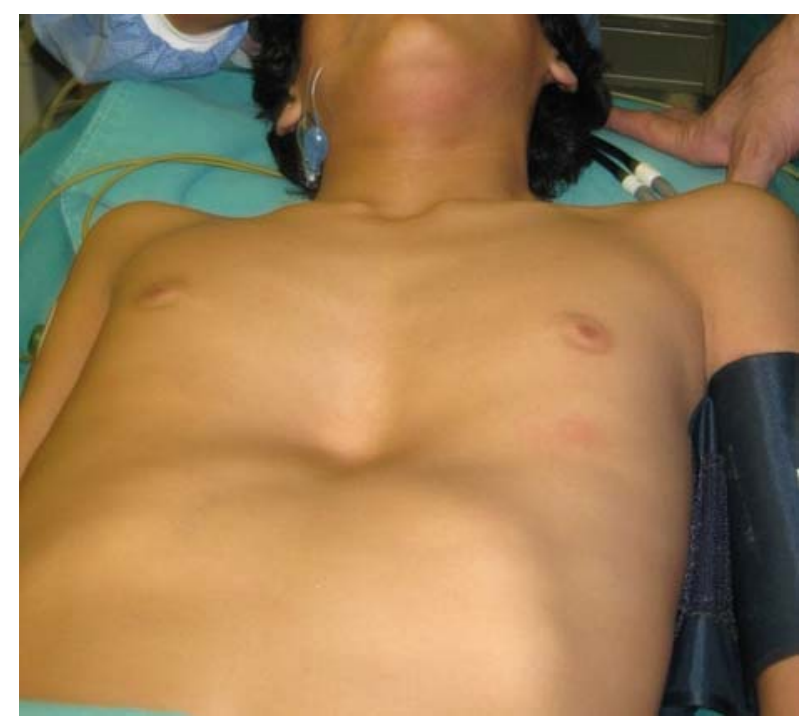

Figura 1. Paciente intraoperatorio con PE simétrico (rango: 4,7 - 6,9) (Figura 2) y estuvo asociado a patología bronco-pulmonar en 4 casos, cardiológico en 4 , recidiva de pectus en 1 y problema psicológico en 1. En la TAC, todos presentaron compresión cardiaca (Tabla 1).

En 8 pacientes se usó un toracoscopio de $5 \mathrm{~mm}$, en 7 pacientes se instaló en el hemitórax derecho y en 3 casos bilateral. A todos los pacientes se les colocó una barra, se emplearon dos estabilizadores fijados con alambre quirúrgico en 7 casos y en 3 con nylon 1 (Figura 3, 4, 5). En 8 pacientes se instaló drenaje pleural cerrado; el tiempo operatorio promedio fue 2,5 horas (2-4 horas). El postoperatorio inmediato se realizó en la unidad de cuidados intensivos en 3 pacientes, en la unidad de cuidados intermedios en 2 pacientes y en sala de hospitalización en 5 pacientes. El catéter epidural

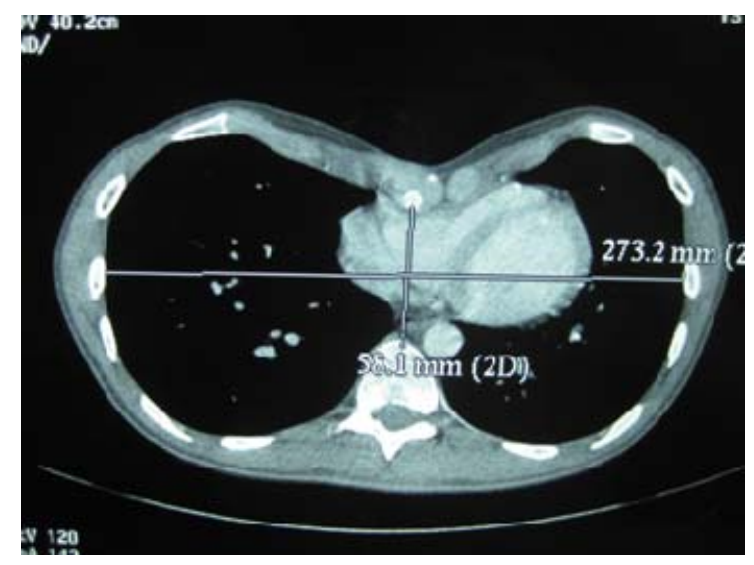

Figura 2. Determinación de Indice de Haller mediante TAC

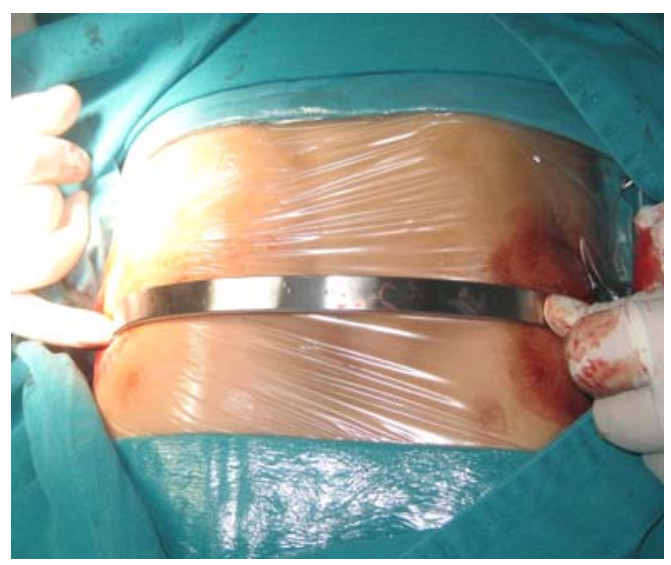

Figura 3. Adecuación de barra metálica. 
Tratamiento del Pectus Excavatum con cirugía minimamente invasiva con la técnica de Donald Nuss.

Tabla 1. Características de los pacientes con Pectus excavatum operados con la técnica mínimamente invasiva.

\begin{tabular}{|c|c|c|c|c|c|c|c|c|}
\hline Paciente & Sexo & Edad (años) & $\begin{array}{l}\text { Indicación } \\
\text { quirúrgica }\end{array}$ & Indice Haller & $\begin{array}{c}\text { Tiempo } \\
\text { Operatorio } \\
\text { (horas) }\end{array}$ & $\begin{array}{c}\text { Días } \\
\text { hospitalización }\end{array}$ & Complicación & $\begin{array}{l}\text { Seguimiento } \\
\text { (meses) }\end{array}$ \\
\hline 1 & M & 6 & Broncopulmonar & 6,9 & 3,0 & 10 & Neumotórax & 14 \\
\hline 2 & $\mathrm{~F}$ & 8 & Cardiológico & 4,8 & 2,3 & 8 & - & 9 \\
\hline 3 & $\mathrm{~F}$ & 8 & Broncopulmonar & 4,7 & 2,5 & 8 & $\begin{array}{c}\text { Fijación } \\
\text { inadecuada de } \\
\text { la barra }\end{array}$ & 4 \\
\hline 4 & M & 15 & Cardiológico & 5,0 & 2,5 & 5 & - & 7 \\
\hline 5 & M & 14 & Cardiológico & 4,9 & 2,5 & 5 & - & 7 \\
\hline 6 & M & 7 & $\begin{array}{l}\text { Recidiva post } \\
\text { Ravich }\end{array}$ & 5,6 & 2,0 & 5 & - & 7 \\
\hline 7 & M & 15 & Broncopulmonar & 4,7 & 2,3 & 7 & - & 4 \\
\hline 8 & M & 14 & Broncopulmonar & 4,9 & 2,0 & 7 & - & 3 \\
\hline 9 & M & 22 & Psicológico & 4,9 & 4,0 & 12 & Neumotórax & 2 \\
\hline 10 & M & 6 & Cardiológico & 5,2 & 2,0 & 6 & - & 2 \\
\hline TOTAL & & $11,5 \pm 5,29$ & & $5,16 \pm 0,67$ & $2,51 \pm 0,61$ & $7,3 \pm 2,31$ & & $5,9 \pm 3,72$ \\
\hline
\end{tabular}

Todos los pacientes tuvieron compresión crdiaca en la Tomografía axial computarizada.

La condiición clínica en el seguimiento fue asintomático en la totalidad de los pacientes.

Todos los pacientes tenían satisfacción de su imagen corporal, en el postoperatorio.

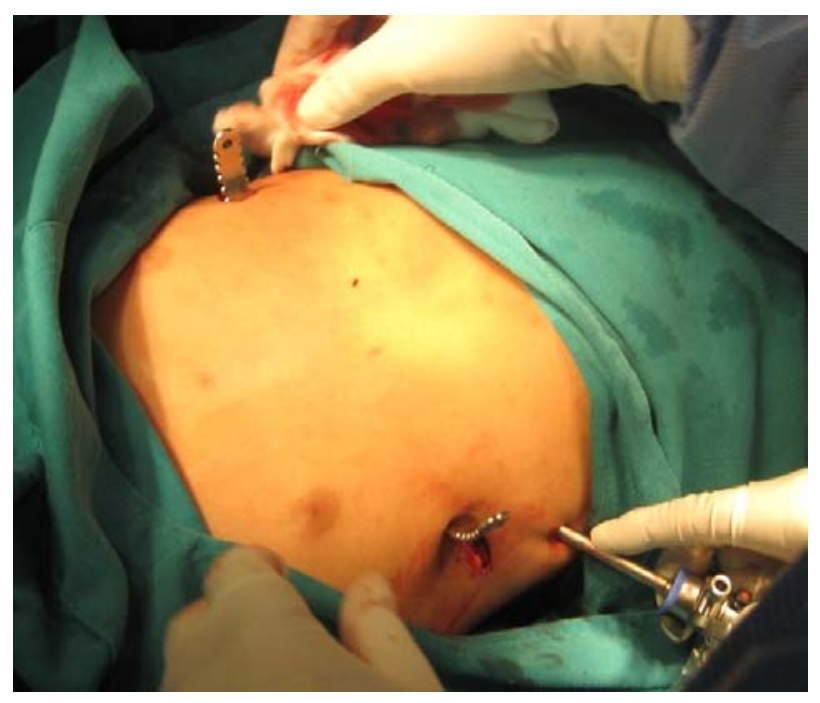

Figura 4. Barra metálica retroesternal en posición cóncava y toracoscopio

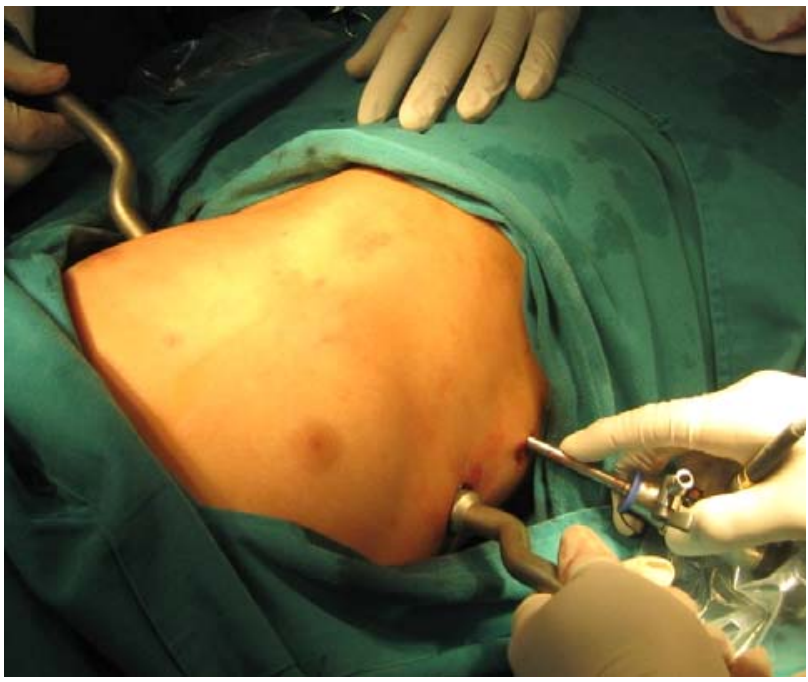

Figura 5. Barra metálica retroesternal en posición convexa, con volteadores y toracoscopio. 


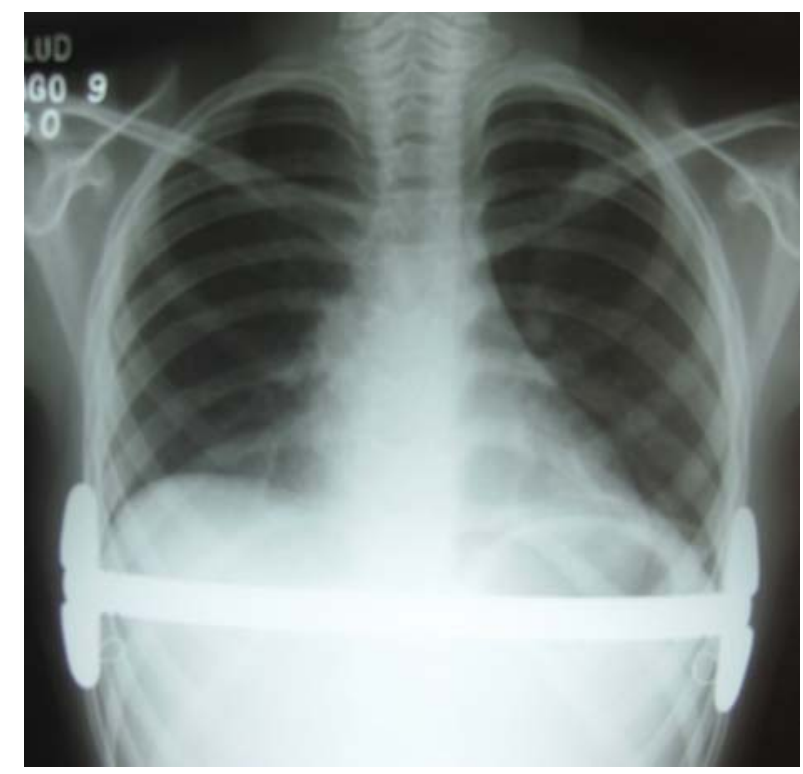

Figura 6. Radiografía: posición final de barra metálica y estabilizadores.

se retiró al tercer día y el drenaje pleural a los 2 días, realizándose control radiológico al tercer día en todos los pacientes (Figura 6).

La estancia hospitalaria promedio fue 7 días. En el Hospital Víctor Lazarte fue 9 días, en el Hospital de Huancayo 5 días y en el Hospital Rebagliati 7 días. No se presentaron complicaciones intraoperatorias; las complicaciones hospitalarias fueron: neumotórax en dos pacientes, un paciente presentó fijación inadecuada del estabilizador del lado izquierdo. En el seguimiento, no se presentaron complicaciones tardías y todos los pacientes estaban clinicamente asintomáticos y presentaron satisfacción de su imagen corporal (Figura 7).

\section{DISCUSIÓN}

El estudio tiene limitaciones, por ser una serie inicial, pequeña, realizada con diferentes grupos quirúrgicos, a pesar que el autor ha participado en todas las intervenciones quirúrgicas, no tiene grupo de control o comparativo y puede existir sesgo.

Ravitch en 1949 introdujo el tratamiento quirúrgico del PE, mediante la técnica de condro-esternoplastía e incisión amplia, vertical o transversal, y Welch en 1958 la mejoró, conservando el pericondrio; Nuss en 1998 describió la corrección quirúrgica en forma mínimamente invasiva, con incisiones laterales pequeñas, empleando una barra de acero colocada en forma retro esternal por vía toracoscópica, sin resección de tejido.

La indicación quirúrgica se ha basado en el IH mayor de 3,25 asociado a una de patologías como: enfermedad bronco-pulmonar en 4 pacientes, cardiológico en 4 , psicológico en 1 y cirugía post Ravitch en 1; similar a otros reportes $(1,2,9)$.

La técnica minimamente invasiva fue diseñada para pacientes menores de 15 años, sin embargo,
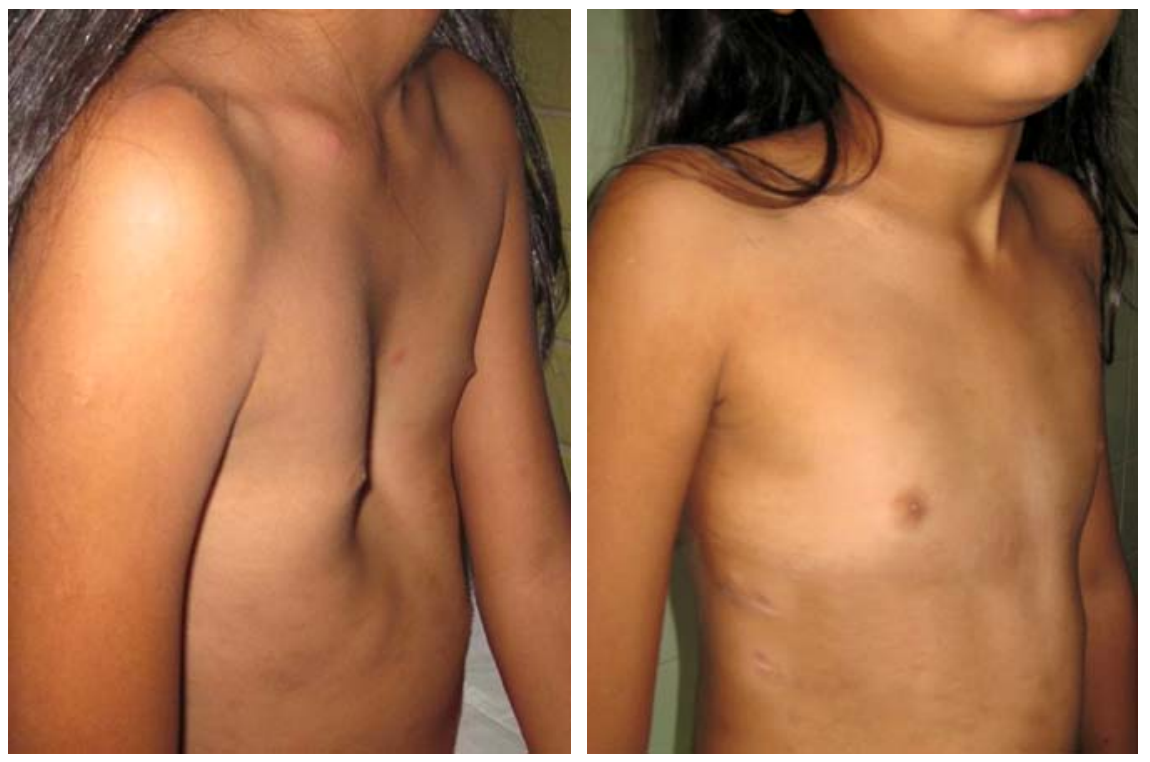

Figura 7. Paciente con PE, pre-operatorio y 4 meses de post-operatorio. 
actualmente se emplea en pacientes adultos, e inclusive se ha reportado en un paciente de 2 meses; el rango en nuestra serie fue de 6 a 22 años. Se considera que la edad no debe ser contraindicación quirúrgica (11-15).

Se han realizado modificaciones a la técnica original, como el empleo de toracoscopio de rutina, el uso de un introductor o disector especial de Kelly para la disección del túnel retro-esternal, el uso de estabilizador lateral y sutura con alambre de acero inoxidable o polydioxano, alrededor de la barra y debajo de la costilla para prevenir el desplazamiento de la barra $(6,11)$. Otros autores han reportado variaciones, como la inserción de la barra por vía extra-pleural con toracoscopio intrapleural, el no empleo de estabilizadores y ni toroacoscopio $(1,9,16)$. Nosotros hemos utilizamos una barra por paciente, con dos estabilizadores. En adultos la mayoría de centros instalan dos barras; así mismo se puede emplear un solo estabilizador.

Las complicaciones reportadas son: neumotórax, hemotórax, perforación cardiaca, ruptura hepática, derrame pleural, derrame pericardico, dehiscencia del alambre de fijación, ruptura muscular, atelectasia, infección de la barra, rotación de la barra, erosión de la piel, reacción alérgica, seroma, granuloma (17-19). En nuestro estudio ocurrió neumotórax en dos casos y fijación inadecuada del estabilizador en el lado izquierdo en un caso. Dos pacientes presentaron dolor intenso, uno debido a que no se siguió el protocolo de terapia del dolor y en el otro de 22 años, probablemente por la rigidez de los tejidos. La estancia hospitalaria en nuestra serie fue de 7 días, similar a otros centros (20).

El costo de la barra y el retardo administrativo para la adquisición de la barra, hacen que muchos pacientes no tengan acceso a esta cirugía; mayor problema tienen los pacientes que no tiene seguro social. La barra es cara y en muchos países no permiten su importación y han comenzado a fabricar sus propias barras de buena calidad y accesibles $(9,15)$.

En el seguimiento los pacientes no presentan la sintomatología inicial, han recuperado su autoestima y anatómicamente existe una mejoría evidente. Los pacientes han tolerado bien y los resultados fueron satisfactorios, similar a otras series $(21,22)$.

Las ventajas de la técnica de Nuss en relación con las técnicas convencionales, están bien establecidas por la simplicidad, ampliación de la indicación quirúrgica a adultos, menor tiempo operatorio, mínimo de complicaciones inmediatas y tardías, disminución del tiempo de retorno a sus actividades y los resultados estéticos excelentes, de tal manera que actualmente se ha constituido en el tratamiento de elección para el PE y ha sido aceptado en la mayoría de los centros médicos mundiales y con seguridad se convertirá en la mejor alternativa quirúrgica a nivel mundial a medida que la curva de aprendizaje mejore $(5,6,8,10,21,22)$.

Los pacientes con PE deben tener la alternativa de las bondades de esta técnica, de tal manera que a un país como el nuestro, la adquisición de la barra debe ser accesible o de lo contrario evaluar otras alternativas de adquisición o fabricación.

\section{Correspondencia}

Mario Lopez Carranza.

Av Mansiche 796

Trujillo-Peru.

Telefono: 51-949664400

Correo electrónico: mariolopezcar@yahoo.es

\section{REFERENCIASBIBLIOGRÁFICAS}

1. Nuss D, Kelly R Croitoru D, Katz M. A 10-year review of a minimally invasive technique for the correction of Pectus Excavatum. J Pediatr Surg 1998; 33: 545-52.

2. Varela P. Pectus excavatum: Historia y propuestas actuales para el estudio y tratamiento. Rev Med Clin Condes 2009; 20(6): 769-775.

3. Haller J, Kramer S, Lietman S. Use of CT scans in selection of patients for pectus excavatum surgery: a preliminary report. J Pediatr Surg 1987; 22: 904-6.

4. Pizon J, González G, Pérez A, Martínez A, Conde J, Benito L. Corrección de la recidiva del pectus excavatum postRavitch con la técnica de Nuss. Cir Pediatr 2009; 22:77-80.

5. Ansíela J, Arancibia I, Ramírez J, Venturelli F, Bertan C. Videotoracoscopia en el tratamiento del pectus excavatum. Cuad Cir. 2005; 19:60-65.

6. Croitoru D, Kelly R, Goretsky M, Lawson L, Swovelend B, Nuss D. Experience and modification update for the minimally invasive Nuss tecnique for pectus excavatum repair in 303 patients. J Pediatr Surg 2002; 37(3): 437-445.

7. Nuss D. Recent experiencs with minimally invasive pectus excavatum repair "nuss procedure”. Jpn J Thorac Cardiovas Surg. 2005; 53(7):338-344.

8. Nuss D. Minimally invasive surgical repair of pectus excavatum. Semin Pediatr Surg 2008; 17: 209-17.

9. Bohosiewicz J, Kudela G, Izwaryn U. The Nuss procedure for the correction of pectus excavatum. Polski Przeglad Chirurgiczny 2009; 81(2):103-112.

10. Pilegaard H, Licht P. Routine use of minimally invasive 
surgery for pectus excavatum in adults. Ann Thorac Surg 2008; 86: 952-7.

11. Aronson D, Bosgraaf R, Van Der Horst C, Ekkelkamp S. Nuss procedure: pediatric surgical solution for adults with pectus excavatum. World J Surg 2007; 31: 26-9.

12. Teh S, Hanna A, Pham T, et al. Minimally invasive repair for pectus excavatum in adults. Ann Thorac Surg 2008; 85: 1914-8.

13. Schalamon J, Pokall S, Windhaber J, Hoellwarth M. Minimally invasive correction of pectus excavatum in adult patients. J Thorac Cardiovasc Surg 2006; 132: 524-9.

14. Krasopoulos G, Dusmet M, Ladas G, Goldstraw P. Nuss procedure improves the quality of life in young male adults with pectus excavatum deformity. Eur J Cardiothorac Surg 2006; 29: 1-5.

15. Prats R, González R, Venturelli R, Lazo R, Santolaya R, Rodríguez P. Corrección mínimamente invasiva (Operación de Nuss) del pectus excavatum en pacientes adultos. Rev Med Chil 2009; 137: 1583-1590.

16. Hernandez H, Varela A, Cordoba M, et al. Videothoracoscopic extrapleural insertion of Walter Lorenz Surgical bar for pectus excavatum for pectus excavatum. JTCS 2003; 126: 2081-82.
17. Shin S, Goretsky M, Kelly Re J, Gustin T, Nuss D. Infectious complications after the Nuss repair in a series of 863 patients. J Pediatr Surg 2007; 42: 87-92.

18. Vegunta R, Pacheco P, Wallace L, Pearl R. Complications associated with the Nuss procedure: continue evolution of the learning curve. Am J Surg 2008; 195: 313-7.

19. Castellani C, Schalamon J, Saxena A, Hoellwarth M. Early complications of the Nuss procedure for pectus excavatum: a prospective study. Pediatr Surg Int 2008; 24: 659-666.

20. Inge T, Owings E, Blewett C, et al. Reduced hospitalizacion cost for patients with pectus excavatum treated using minimally invasive surgery. Surg Endosc 2003: 17 (10):1609-1613.

21. Bardaji C, Maldonado J, Royo Y, Pueyo C, Carrasco R, Barbero L. Nuestra experiencia en el tratamiento del pectus excavatum severo mediante toracoplastia percutanea videoassistida. Nascer e Crescer 2005; 14 (1): 9-14.

22. Park H, Lee S, Lee C, Youm W, Lee K. The Nuss procedure for pectus excavatum: evolution of techniques and early results on 322 patients. Ann Thorac Surg 2004;77: 289-95.

Recibido: 14/03/11

Aceptado para publicación: 15/08/11 